Recent Insights into the Physics of the Sun and Heliosphere:

Highlights from $\mathrm{SOHO}$ and Other Space Missions

IAU Symposium, Vol. 203, 2001

P. Brekke, B. Fleck, and J. B. Gurman eds.

\title{
The Quiet-Sun Variability as Seen by CDS and SUMER
}

\author{
A. Brković \\ Institute of Astronomy, ETH Zentrum, CH-8092 Zürich, Switzerland \\ S. K. Solanki \\ Max-Planck-Institut für Aeronomie, Max-Planck-Str. 2, D-37191 \\ Katlenburg-Lindau, Germany \\ I. Rüedi \\ Physikalisch-Meteorologisches Observatorium Davos, World Radiation \\ Center, CH-7260 Davos Dorf, Switzerland
}

\begin{abstract}
We investigate brightness variations of the quiet Sun using movies obtained with CDS and SUMER time series on SOHO. We compare the variability and relative variability detected with the two instruments and find that SUMER reveals a factor 2-3 higher variability than CDS.
\end{abstract}

\section{Introduction}

Using CDS Brković et al. (2000) found that all parts of the quiet Sun were significantly variable on time-scales longer than $5 \mathrm{~min}$ in the He I $584.3 \AA$ and O V $629.7 \AA$ lines, while the variability in the Mg IX $368.1 \AA$ line was more marginal. The same work showed that the highest relative variability was observed in $\mathrm{O} V$, then in He I and the least one in Mg IX. Now we extend the analysis to the new data obtained simultaneously with CDS and with SUMER.

\section{Observations and data}

Two co-aligned measurements of quiet regions at sun centre were carried out on 23rd April 1997 with the Normal Incidence Spectrometer (NIS) of the Coronal Diagnostic Spectrometer (CDS) (Harrison et al. 1995) and the Solar Ultraviolet Measurements of Emitted Radiation (SUMER) spectrometer (Wilhelm et al. 1995) onboard the SOHO satellite. NIS was in its movie mode, i.e. $90^{\prime \prime} \times 240^{\prime \prime}$ slit with a pixel size of $1.68^{\prime \prime} \times 1.68^{\prime \prime}$. The He I, O V and $\mathrm{Mg}$ IX lines were recorded at a cadence of 15 seconds for durations of 230 and $300 \mathrm{~min}$. SUMER observed simultaneously with CDS at cadences of 19 seconds (1st measurement with the He I 584.3 $\AA$ and O I $1152.1 \AA$ lines) and 34 seconds (2nd measurement with the O V $629.7 \AA$ and C I 1267.6 $\AA$ lines). The SUMER data were obtained with the $1^{\prime \prime} \times 120^{\prime \prime}$ slit, with the pixel size being $1^{\prime \prime} \times 1^{\prime \prime}$. The SUMER slit was kept at a fixed location on the solar surface. 

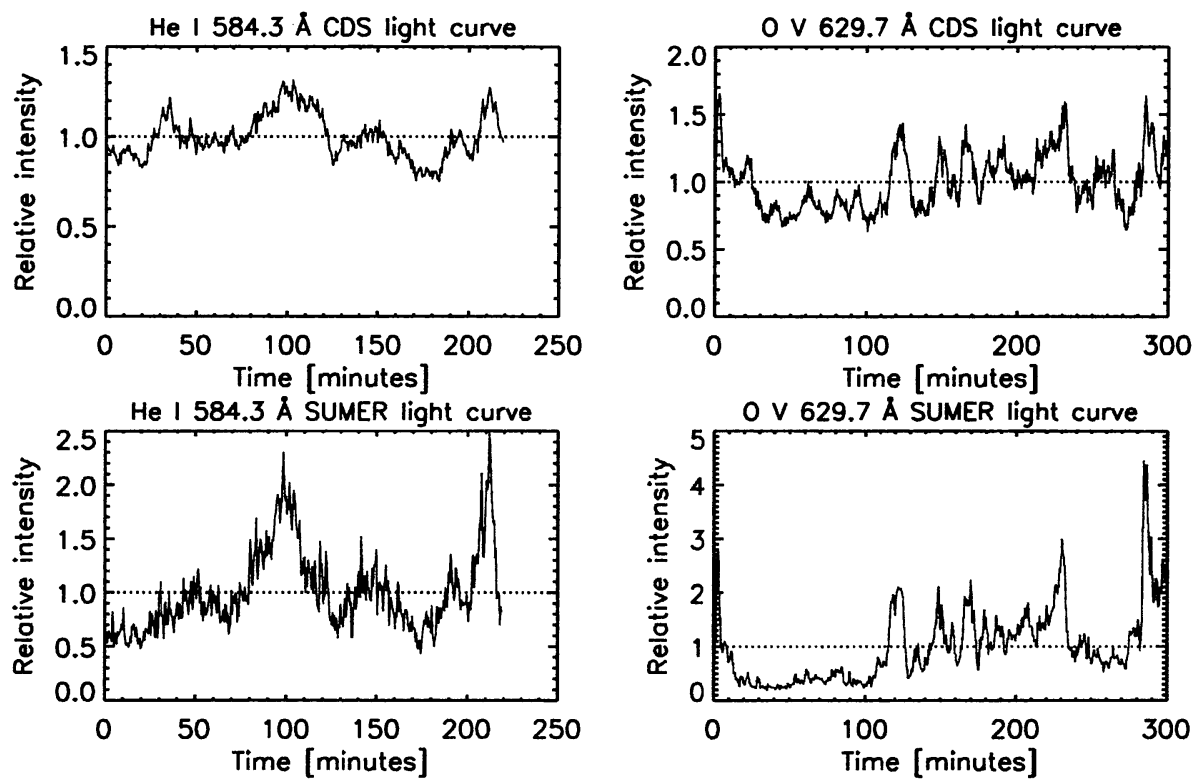

Figure 1. Sample light curves for the He I and O V lines of CDS and SUMER. The vertical axes of the light curve frames show the intensity relative to the averaged intensity (the horizontal dotted lines). Note the difference in scale between the SUMER and CDS light curves.

\section{Analysis}

For each pixel we determined the variability, the temporal average brightness and the ratio of these values (i.e. relative variability) in the same manner as described by Brković et al. (2000). Figure 1 shows examples of light curves for the $\mathrm{He} \mathrm{I}$ and $\mathrm{O} \mathrm{V}$ lines for the same spatial locations. The differences in scales of relative intensities between lines and those between instruments are remarkable. The differences in scales between $\mathrm{He} \mathrm{I}$ and $\mathrm{O} \mathrm{V}$ are of solar origin, while the differences between the instruments have its cause in the higher spatial resolution of SUMER compared to CDS.

Figure 2 shows relative variabilities for the CDS and SUMER lines. The two sets of CDS data were combined since the relative variabilities on time scales of 230 and $300 \mathrm{~min}$ were practically the same for a given line. A comparison of the He I and O V lines shows that the level of relative variability seen by both instruments is higher in $\mathrm{O} \mathrm{V}$ than in $\mathrm{He} \mathrm{I}$, as already cited. It also shows that the observed levels of relative variabilities are roughly a factor of 3 higher in the SUMER than in the CDS measurements. The median variabilities are $9.5 \%$ for He I and $17.5 \%$ for $\mathrm{O} V$ as seen with CDS, while corresponding values as seen with SUMER amount to $23.5 \%$ for He and $55.0 \%$ for O V. In other respects both instruments give similar results, which agree with those of Brković et al. (2000). E.g., we found that the absolute variabilities are well correlated between different lines, the relative variabilities are independent of the time-averaged brightness in all lines and the brightness fluctuations are mainly due to brightness changes 
on time-scales longer than $5 \mathrm{~min}$. Fluctuations in $\mathrm{O}$ I and C I differ in the sense that brightness changes on time-scales $\leq 5 \mathrm{~min}$ are also very significant.
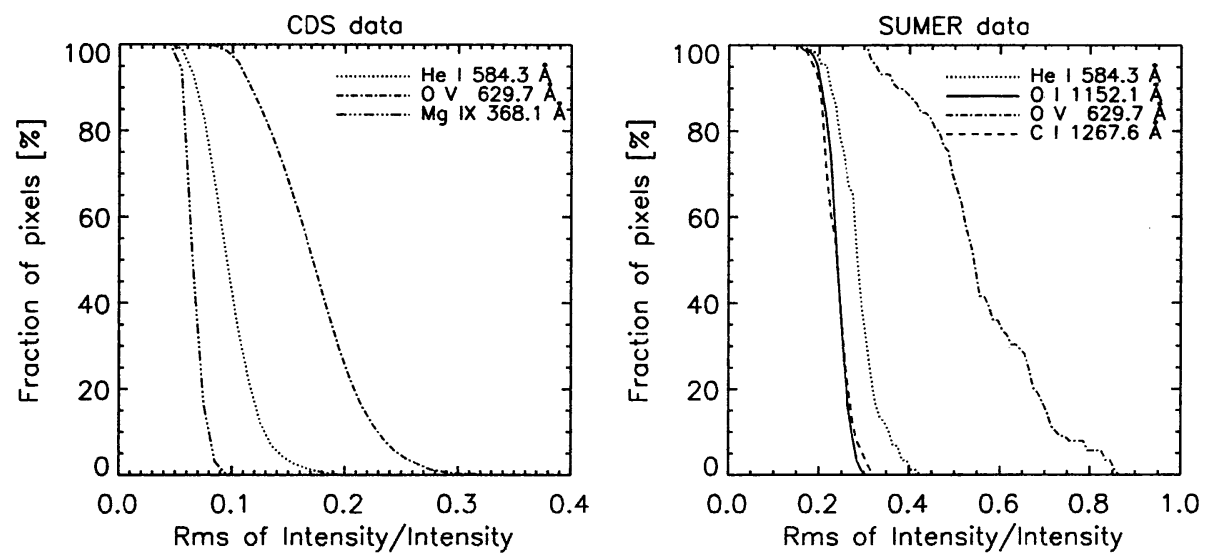

Figure 2. Fraction of points with relative variability lying above the corresponding value on the abscissa for CDS and SUMER lines.

\section{Summary}

The analysis of co-aligned movies of the quiet-Sun centre obtained with CDS and SUMER confirmed the results of Brković et al. (2000). It has also been found that the level of relative variability increases strongly with increasing spatial resolution of the instrument involved.

Acknowledgments. AB thanks U. Schühle, A. Pauluhn and K. Stucki for helping with the SUMER data handling. We would like to thank the CDS and SUMER teams, as well as the SOHO command staff whose help was invaluable in obtaining these observations. SOHO is a mission of international cooperation between ESA and NASA. This work was partly supported by the Swiss National Science Foundation, grant No. 21-45083.95, and by a grant from the ETH-Zürich which is gratefully acknowledged.

\section{References}

Brković, A., Rüedi, I., Solanki, S.K. et al. 2000, A\&A, 353, 1083

Harrison, R.A., Sawyer, E.C., Carter, M.K. et al. 1995, Solar Phys., 162, 233

Wilhelm, K., Curdt, W., Marsch, E. et al. 1995, Solar Phys. 162, 189 